\title{
Memory for Light as a Quantum Process
}

\author{
M. Lobino ${ }^{1}$ C. Kupchak, ${ }^{1}$ E. Figueroa,${ }^{1,2}$ and A. I. Lvovsky ${ }^{1 *}$ \\ ${ }^{1}$ Institute for Quantum Information Science, University of Calgary, Calgary, Alberta T2N 1N4, Canada and \\ 2 Max-Planck-Institut für Quantenoptik, Hans-Kopfermann-Str. 1, 85748 Garching, Germany
}

\begin{abstract}
We report complete characterization of an optical memory based on electromagnetically induced transparency. We recover the superoperator associated with the memory, under two different working conditions, by means of a quantum process tomography technique that involves storage of coherent states and their characterization upon retrieval. In this way, we can predict the quantum state retrieved from the memory for any input, for example, the squeezed vacuum or the Fock state. We employ the acquired superoperator to verify the nonclassicality benchmark for the storage of a Gaussian distributed set of coherent states.
\end{abstract}

PACS numbers: 42.50.Ex, 03.67.-a, 32.80.Qk, 42.50.Dv

Introduction Quantum memory for light is an essential technology for long distance quantum communication [1] and for any future optical quantum information processor. Recently, several experiments have shown the possibility to store and retrieve nonclassical states of light such as the single photon [2, 3], entangled [4] and squeezed vacuum [5, [6] states using coherent interactions with an atomic ensemble.

In order to evaluate the applicability of a quantum memory apparatus for practical quantum communication and computation, it is insufficient to know its performance for specific, however complex, optical states, because in different protocols, different optical states are used for encoding quantum information [1, 7]. Practical applications of memory require answering a more general question: how will an arbitrary quantum state of light be preserved after storage in a memory apparatus?

Here we answer this question by performing complete characterization of the quantum process associated with optical memory based on electromagnetic induced transparency (EIT) 8]. Memory characterization is achieved by storing coherent states (i. e. highly attenuated laser pulses) of different amplitudes and subsequently measuring the quantum states of the retrieved pulses. Based on the acquired information, the retrieved state for any arbitrary input can be predicted and additionally, any theoretical benchmark on quantum memory performance can be readily verified.

Coherent state quantum process tomography We can define complete characterization of an optical quantum memory as the ability to predict the retrieved quantum state $\hat{\mathcal{E}}(\hat{\rho})$ when the stored input state $\hat{\rho}$ is known. This is a particular case of the quantum "black box" problem, which is approached through a procedure called quantum process tomography (QPT) [9]. QPT is based on the fact that every quantum process (in our case, optical memory) is a linear map on the linear space $\mathbb{L}(\mathbb{H})$ of density matrices over the Hilbert space $\mathbb{H}$ on which the process is defined. The associated process can thus be characterized by constructing a spanning set of "probe" states in $\mathbb{L}(\mathbb{H})$ and subjecting each of them to the action of the quantum "black box". If we measure the process output $\hat{\mathcal{E}}\left(\hat{\rho}_{i}\right)$ for each member $\hat{\rho}_{i}$ of this spanning set, we can calculate the process output for any other state $\hat{\rho}=\sum_{i} a_{i} \hat{\rho}_{i}$ according to

$$
\hat{\mathcal{E}}(\hat{\rho})=\sum_{i} a_{i} \hat{\mathcal{E}}\left(\hat{\rho}_{i}\right)
$$

The challenge associated with this approach is the construction of the appropriate spanning set, given the infinite dimension of the optical Hilbert space and the lack of techniques for universal optical state preparation. For this reason, characterizing memory for light, that is not limited to the qubit subspace, is much more difficult than memory for superconducting qubits, which has been reported recently [10]. Our group has recently developed a process characterization technique that overcomes these challenges [11]. Any density matrix $\hat{\rho}$ of a quantum optical state can be written as a linear combination of density matrices of coherent states $|\alpha\rangle$ according to the optical equivalence theorem

$$
\hat{\rho}=2 \int P_{\hat{\rho}}(\alpha)|\alpha\rangle\langle\alpha| d^{2} \alpha,
$$

where $P_{\hat{\rho}}(\alpha)$ is the state's Glauber-Sudarshan P-function and the integration is performed over the entire complex plane. Although the P-function is generally highly

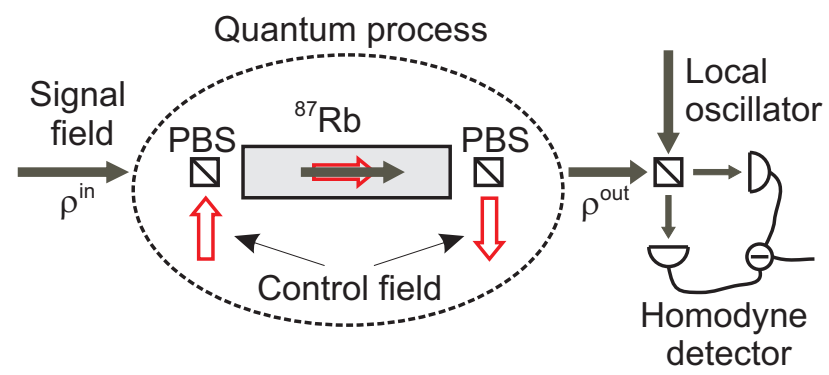

FIG. 1: (color online). Schematic of the experimental setup used to characterize the process associated with the quantum memory. PBS, polarizing beam splitter. 
singular, any quantum state can be arbitrarily well approximated by a state with an infinitely smooth, rapidly decreasing P-function 12]. Therefore, by measuring how the process affects coherent states, one can predict its effect on any other state. The advantage of such approach (which we call coherent-state quantum process tomography or csQPT) is that it permits complete process reconstruction using a set of "probe" states that are readily available from a laser.

Experimental setup We performed csQPT on optical memory [6] realized in a warm rubidium vapor by means of electromagnetically-induced transparency (Fig. 1). The atoms are ${ }^{87} \mathrm{Rb}$ and the vapor temperature is kept constant at $65^{\circ} \mathrm{C}$.

The signal field is resonant with the $\left|{ }^{5} S_{1 / 2}, F=1\right\rangle \leftrightarrow$ $\left|{ }^{5} P_{1 / 2}, F=1\right\rangle$ transition at $795 \mathrm{~nm}$ and is produced by a continuous-wave Ti:Sapphire laser. An external cavity diode laser, phase locked at $6834.68 \mathrm{MHz}$ to the signal laser 13 serves as the EIT control field source, and is resonant with the $\left|{ }^{5} S_{1 / 2}, F=2\right\rangle \leftrightarrow\left|{ }^{5} P_{1 / 2}, F=1\right\rangle$ transition. The fields are red detuned from resonance by 630 $\mathrm{MHz}$ in order to improve the storage efficiency. The control field power is $5 \mathrm{~mW}$ and the beam spatial profile is mode matched with the signal beam to a waist of $0.6 \mathrm{~mm}$ inside the rubidium cell. Signal and control fields are orthogonally polarized; they are mixed and separated using polarizing beam splitters.

The two photon detuning $\Delta_{2}$ between the signal and control fields is modified by varying the frequency of the control field laser through the phase lock circuit, while an acousto-optical modulator (AOM) is used to switch on and off the control field intensity. We analyzed two different operative conditions characterized by $\Delta_{2}=0$ and $0.54 \mathrm{MHz}$.

The input pulse is obtained by chopping the continuous-wave signal beam via an AOM to produce $1 \mu$ s pulses [Fig. 2(c)] with a $100 \mathrm{kHz}$ repetition rate. A second AOM is used to compensate for the frequency shift generated by the first. Transfer of the light state into the atomic ground state superposition (atomic spin wave) is accomplished by switching the control field off for the storage duration of $\tau=1 \mu$ s when the input pulse is inside the rubidium cell.

We performed full state reconstruction of both the input and retrieved fields by time domain homodyne tomography [14]. A part of the Ti:Sapphire laser beam serves as a local oscillator for homodyne detection; while its phase is scanned via a piezoelectric transducer, the homodyne current is recorded with an oscilloscope. For every state, 50000 samples of phase and quadrature are measured and processed by the maximum likelihood algorithm [15, 16], estimating the state density matrix in the Fock basis.

Tomography of quantum memory In order to determine the coherent state mapping necessary for reconstructing the process, we measured 10 different coherent

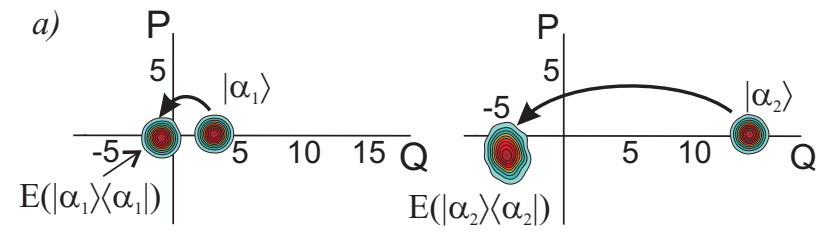

b)
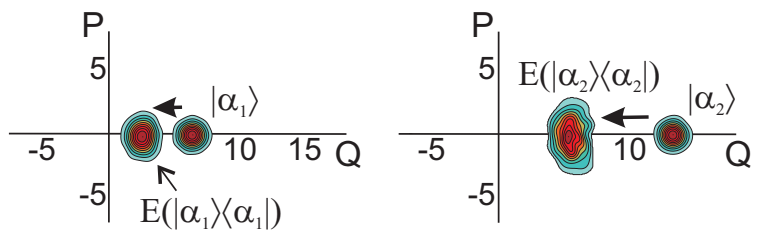

c)

d)
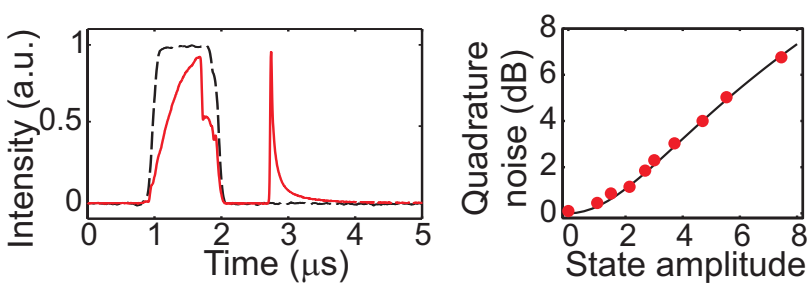

FIG. 2: (color online). Wigner functions of input coherent states with two different amplitudes and the corresponding retrieved states $\hat{\mathcal{E}}\left(\left|\alpha_{1}\right\rangle\left\langle\alpha_{1}\right|\right)$ and $\hat{\mathcal{E}}\left(\left|\alpha_{2}\right\rangle\left\langle\alpha_{2}\right|\right)$. The input state amplitudes are $\alpha_{1}=2.3, \alpha_{2}=10.3$ (a) and $\alpha_{1}=4.5$, $\alpha_{2}=10.9$ (b). Two-photon detunings between the control and signal fields are $0.54 \mathrm{MHz}$ (a) and $0 \mathrm{MHz}$ (b). Input pulse (black dashed line) and retrieved light (red solid line) (c). Variance of the phase quadrature as a function of the retrieved state amplitude $(\mathrm{d})$.

states $\left|\alpha_{i}\right\rangle$ with mean photon numbers ranging from 0 to 285 along with their corresponding retrieved states $\hat{\mathcal{E}}\left(\left|\alpha_{i}\right\rangle\left\langle\alpha_{i}\right|\right)$ [Fig. 2(a) and (b)]. Subsequently, we applied polynomial interpolation to determine the value of $\hat{\mathcal{E}}(|\alpha\rangle\langle\alpha|)$ for any value of $\alpha$ in the range 0 to 16.9 . Performing tomographic reconstruction for these highly displaced states requires good phase stability between the signal and local oscillator. Phase fluctuations produce an artefact in the reconstruction in the form of amplitude dependent increase in the phase quadrature variance. In our measurements, the reconstructed input states $\left|\alpha_{i}\right\rangle$ resemble theoretical coherent states with a fidelity higher than 0.999 for mean photon values up to 150 [Fig. 2](a) and (b)].

By inspecting the Wigner functions of the input and retrieved states, one can clearly notice the detrimental effects of the memory. First, there is attenuation of the amplitude by a factor of $0.41 \pm 0.01$ for the signal field in two-photon resonance with the control, which increases to a factor of $0.33 \pm 0.02$ when a two-photon detuning of $\Delta_{2}=0.54 \mathrm{MHz}$ is introduced. This corresponds to a mean photon number attenuation by factors of $0.17 \pm 0.02$ and $0.09 \pm 0.01$, respectively. Note that in the case of nonzero two-photon detuning, the attenuation is greater than the factor of 0.14 obtained in classical intensity mea- 


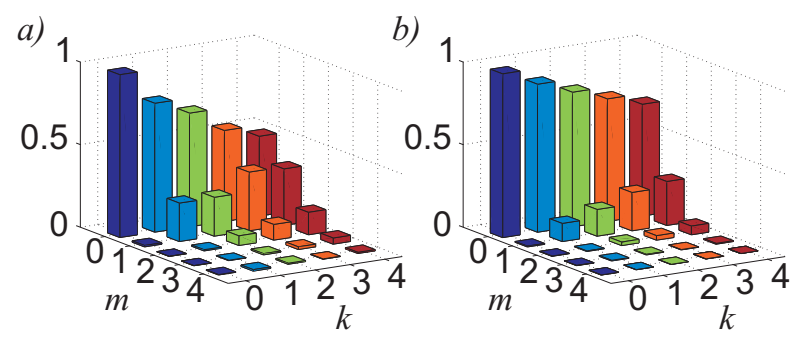

FIG. 3: (color online). The diagonal elements of the process tensor $\chi_{k k}^{m m}$, measured by csQPT in the Fock basis for $\Delta_{2}=$ 0 (a) and $0.54 \mathrm{MHz}$ (b).

surement [Fig. 2(c)]. This is because the temporal mode of the retrieved state is slightly chirped, and could not be perfectly matched to the mode of the local oscillator.

Second, retrieved coherent states experience an increase in the phase quadrature variance that depends quadratically on the state amplitude. This effect produces an ellipticity in the retrieved state Wigner function (Fig. 2(a) and (b)] and can be attributed to the noise in the phase lock between the signal and control lasers [13]. Fluctuations $\Delta \phi$ of the relative phases between the two interacting fields randomize the phase of the retrieved signal field with respect to the local oscillator. Assuming a Gaussian distribution for $\Delta \phi$ with zero mean and variance $\sigma_{\phi}^{2}$ the variance of the phase quadrature can be expressed as:

$$
\sigma_{q}^{2}=\frac{1}{2}+\frac{q_{0}^{2}}{2}\left(1-e^{2 \sigma_{\phi}^{2}}\right)
$$

where $q_{0}$ is the mean amplitude. We fit our experimental data with $\mathrm{Eq} 3$ and estimate an $11^{\circ}$ standard deviation for $\Delta \phi$ [Fig. 2(d)], in agreement with independent estimates [13].

The third detrimental effect preventing the atomic ensemble from behaving as a perfect memory is the population exchange between atomic ground states [17, 18]. Besides limiting the memory lifetime, this exchange generates spontaneously emitted photons in the signal field mode adding an extra noise that thermalizes the stored light by increasing the quadrature variance independently of the input amplitude and phase. We measured the extra noise from the quadrature variance of retrieved vacuum states $\hat{\mathcal{E}}(|0\rangle\langle 0|)$ and found it to equal $0.185 \mathrm{~dB}$ when both fields were tuned exactly at the two photon resonance, which corresponds to the mean photon number in the retrieved mode equal to $\bar{n}=\operatorname{Tr}[\hat{n} \hat{\mathcal{E}}(|0\rangle\langle 0|)]=$ 0.022. This noise is reduced to $0.05 \mathrm{~dB}$ (corresponding to $\bar{n}=0.005$ ) in the presence of two photon detuning. For this reason, it is beneficial to implement storage of squeezed light in the presence of two-photon detuning, in spite of higher losses.

In the presence of the two-photon detuning, the evolution of the atomic ground state superposition brings about a phase shift of the retrieved state with respect to the input by $2 \pi \Delta_{2} \tau=200^{\circ}$ as is visible in Fig. 2 (a).

Based on the information collected from the storage of coherent states, we reconstruct the memory process in the $\chi$-matrix representation, defined by [19, 20]

$$
\hat{\mathcal{E}}(\hat{\rho})=\sum_{k, l, m, n} \chi_{k, l}^{n, m} A_{l, n} \hat{\rho} A_{m, k},
$$

where $\chi_{k, l}^{n, m}$ is the rank 4 tensor comprising full information about the process and $A_{i, j}$ is a set of operators that form a basis in the space of operators on $\mathbb{H}$. Since $\mathbb{H}$ is the Hilbert space associated with an electromagnetic oscillator, it is convenient to choose $A_{i, j}=|i\rangle\langle j|$, where $|i\rangle$ and $|j\rangle$ are the photon number states. The details of calculating the process tensor are described elsewhere [11]; Fig. 3 displays the diagonal subset $\chi_{k, k}^{m, m}$ of the process tensor elements.

Performance tests In order to verify the accuracy of our process reconstruction, we have used it to calculate the effect of storage on squeezed vacuum with $\Delta_{2}=0.54$
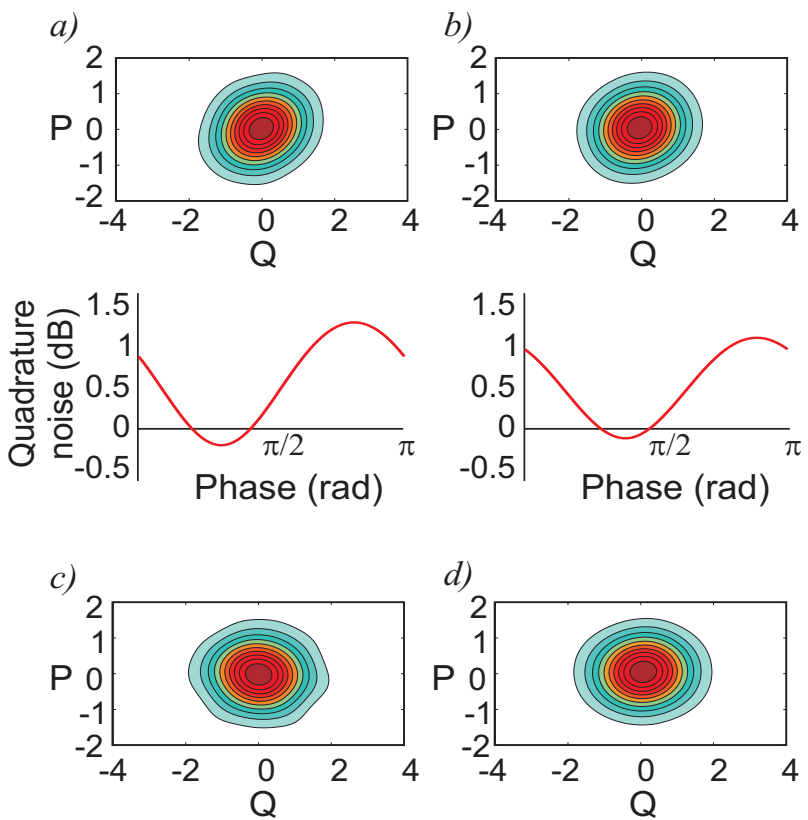

d)
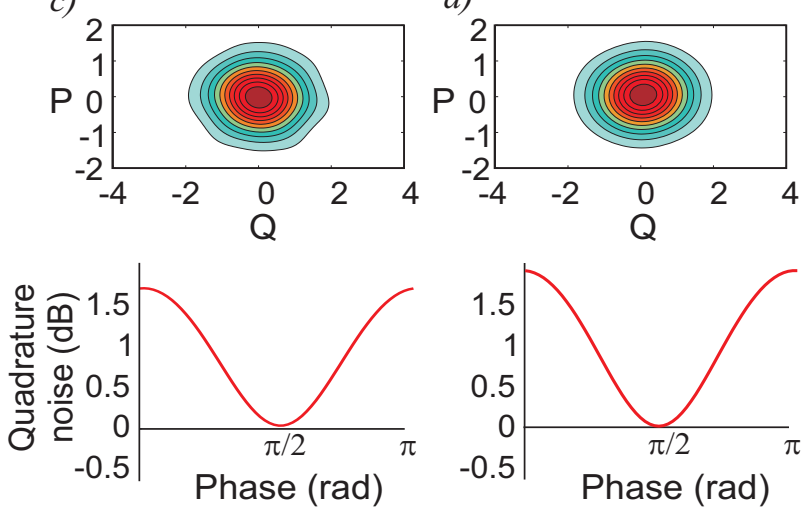

FIG. 4: (color online). Comparison of the experimentally measured squeezed vacuum states retrieved from the quantum memory and those predicted with csQPT. For each case, the Wigner function and the quadrature variance as a function of the local oscillator phase are shown. (a), Experimental measurement [6] with $\Delta_{2}=0.54 \mathrm{MHz}$. (b), Prediction with $\Delta_{2}=0.54 \mathrm{MHz}$. (c), Experimental measurement with $\Delta_{2}=$ 0 . (d), Prediction with $\Delta_{2}=0$. 
$\mathrm{MHz}$, as studied in a recent experiment of our group [ 6], and with $\Delta_{2}=0 \mathrm{MHz}$. We applied the superoperator tensor measured with csQPT to the squeezed vacuum produced by a subthreshold optical parametric amplifier with a noise reduction in the squeezed quadrature of $-1.86 \mathrm{~dB}$ and noise amplification in the orthogonal quadrature of $5.38 \mathrm{~dB}$ (i.e. the same state as used as the memory input in Ref. [6]). In this way, we obtained a prediction for the state retrieved from the memory, which we then compared with the results of direct experiments. This comparison yields quantum mechanical fidelities of $0.9959 \pm 0.0002$ and $0.9929 \pm 0.0002$ for the two-photon detunings of $\Delta_{2}=0.54 \mathrm{MHz}$ and $\Delta_{2}=0$ respectively (Fig. (4).

As discussed above, zero detuning warrants lower losses (thus higher amplitude of the noise variance) and no phase rotation, but higher excess noise (thus no squeezing in the retrieved state). Nevertheless the two photon resonant configuration offers a better fidelity if the single photon state is stored [2, 3], as evidenced by comparing the superoperator element $\chi_{1,1}^{1,1}$ of Fig. 3 (a) and (b).

In addition to the ability to predict the output of the memory for any input state, our procedure can be used to estimate the performance of the memory according to any available benchmark. As an example, we analyze the performance of our memory with respect to the classical limit on average fidelity associated with the storage of coherent states with amplitudes distributed in phase space according to a Gaussian function of width $1 / \lambda[21]$. This limit as a function of $\lambda$ is given by:

$F(\lambda)=2 \lambda \int_{0}^{+\infty} \exp \left(-\lambda \alpha^{2}\right)\langle\alpha|\hat{\mathcal{E}}(|\alpha\rangle\langle\alpha|)| \alpha\rangle \alpha d \alpha \leq \frac{1+\lambda}{2+\lambda}$.

From csQPT data, we evaluate the average fidelity associated with our memory for both values of $\Delta_{2}$ (Fig. 河). Both configurations show nonclassical behavior. The higher value of average fidelity correspond to $\Delta_{2}=0$ and is explained by a higher storage efficiency.

Conclusion In summary, we have demonstrated complete characterization of an EIT-based quantum memory by csQPT. This procedure allows one to predict the effect of the memory on an arbitrary quantum-optical state, and thus provides the "specification sheet" of quantummemory devices for future applications in quantum information technology. Furthermore, our results offer insights into the detrimental effects that affect the storage performance and provide important feedback for the device optimization. We anticipate this procedure to become standard in evaluating the suitability of a memory apparatus for practical quantum telecommunication networks.

Acknowledgements This work was supported by NSERC, iCORE, CFI, AIF, QuantumWorks, iCORE
(C.K.) and CIFAR (A.L.).

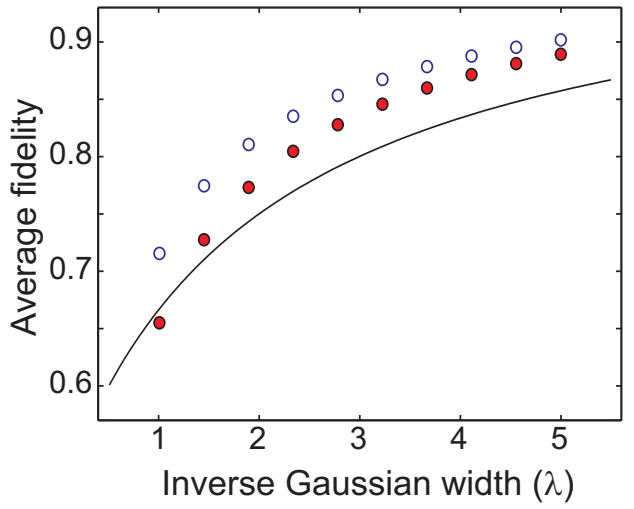

FIG. 5: (color online). Average fidelity of the quantum memory for a Gaussian distributed set of coherent states. Blue empty (red filled) dots show the average fidelity calculated from the csQPT experimental data for $\Delta_{2}=0(0.54 \mathrm{MHz})$. The experimental uncertainty is 0.0002 . The solid line shows the classical limit [21].

* Electronic address: lvov@ucalgary.ca

[1] L. M. Duan, M. D. Lukin, J. I. Cirac, and P. L Zoller, Nature 414, 413 (2001).

[2] T. Chanelere et al., Nature 438, 833 (2006).

[3] M. D. Eisaman et al., Nature 438, 837 (2006).

[4] K. S. Choi, H. Deng, J. Laurat, and H. J. Kimble, Nature 452, 67 (2008).

[5] K. Honda et al., Phys. Rev. Lett. 100, 093601 (2008).

[6] J. Appel et al., Phys. Rev. Lett. 100, 093602 (2008).

[7] S. Pirandola, S. Mancini, S. Lloyd, and S. L. Braunstein, Nature Phys. 4, 726 (2008).

[8] M. Fleischhauer, A. Imamoglu, and J. P. Marangos, Rev. Mod. Phys. 77, 633 (2005).

[9] M. Mohseni, A. T. Rezakhani, and D. A. Lidar, Phys. Rev. A 77, 032322 (2008).

[10] M. Neeley, Nature Physics 4, 523 (2008).

[11] M. Lobino et al., Science 322, 563 (2008).

[12] J. R. Klauder, Phys. Rev. Lett. 16, 534 (1966).

[13] J. Appel, A. MacRae, and A. I. Lvovsky, arXiv:0809.3607

[14] A. I. Lvovsky, and M. G. Raymer, Rev. Mod. Phys. (to be published).

[15] A. I. Lvovsky, J. Opt. B: Quantum and Semiclassical Optics 6, S556 (2004)

[16] J. Řeháček, Z. Hradil, E. Knill, and A. I. Lvovsky, Phys. Rev. A 75, 042108 (2007).

[17] G. Hétet et al., Phys. Rev. A 77, 012323 (2008).

[18] E. Figueroa et al., New J. Phys. (to be published).

[19] M. Nielsen and I. L. Chuang, Quantum Computation and Quantum Information, (Cambridge University Press, Cambridge 2000).

[20] I. L. Chuang, and M. A. Nielsen, J. Mod. Opt. 44, 2455 (1997).

[21] K. Hammerer, M. M. Wolf, E. S. Polzik, and J. I. Cirac, Phys. Rev. Lett. 94, 150503 (2005). 